\title{
An Empirical Study on a Meme-based CET 4 Writing Teaching Model
}

\author{
Wang Jin \\ School of English, Tianjin University Renai College, Tianjin, China, 300143 \\ ilikenine@sina.com
}

Keywords: CET 4 writing, meme, corpus, teaching model.

\begin{abstract}
This paper utilizes meme theory as reference in order to rethink about CET 4 writing teaching pattern. As an essential part of cultural replication factors, language has its own rules in reproduction and transmission. Those laws that language obeys can be properly applied to writing teaching process. Three major questions will be discussed in this paper: 1 . Can meme-based CET 4 writing teaching model effectively benefit non-English major students in CET 4 writing part? 2. In what particular aspects can meme-based CET 4 writing teaching model help improve students' writing level? 3. Are there any limitations and problems existing in meme-based CET 4 writing teaching model?
\end{abstract}

\section{英语四级写作教学模式改革 \\ 基于语料库的模因教学模式实证研究}

\author{
王巾 \\ 天津大学仁爱学院英语系, 天津, 中国 \\ ilikenine@sina.com
}

关键词：四级写作; 模因; 语料库; 教学模式

中文摘要. 本文借鉴模因理论对基于大学英语四级的写作教学模式进行重新研究和探讨, 将 语言作为一种重要的文化复制因子这一特征以及它在复制传播中的规律应用到写作教学过程 中, 探讨模因式英语教学模式对非英语专业学生的四级写作的影响及以下三个主要问题: (1) 与本校英语教师带有个人习惯和特点的传统四级写作的教学模式相比, 模因式教学模式能否 帮助学生提高四级写作水平？（2）模因式教学模式能够在哪些方面帮助学生提高写作能力?

(3) 模因式教学模式是否能被本校学生接受, 此模式有何局限性以及在具体实施过程中存在 哪些问题?

\section{1. 引言}

大学英语四级考试是由教育部高等教育司主办的一项标准化的全国性的教学考试, 考试 主体是所有修完大学英语课程的非英语专业大学生。笔者通过调查发现, 在四级考试题型中, 学生的写作部分是最薄弱的环节, 成绩普遍偏低, 极大地制约了考生的整体成绩水平。作为 语言输出的重要组成部分, 写作反应了学生的语言知识吸收和应用的双重能力, 提高写作成 绩和水平也已成为英语教学中的重要目标之一。 
经过调查笔者发现, 在本校的英语教学课程当中, 针对非英语专业学生的写作指导练习 甚少, 并且每一位教师在讲解四级考试写作时的效果差强人意。这主要源自以下几个原因: 首先, 教师的讲授方式过于简易。主要体现在教师只针对作文题目进行简单的翻译和理解, 却并没有展开对话题进行具体阐述过程的针对性练习。其次, 教师的教学方式过于程式化。 目前写作课的普遍形式是由老师课上布置作文, 学生课下写作文, 然而老师在课下并没有任 何跟进, 也没有针对学生在词语、句式等表达方面进行的练习和巩固工作。上述常见的写作 教学形式并不能有效提高学生的写作能力, 学生也很难达到四级写作的水平。

\section{2. 理论依据和研究目的}

“模因” 的概念最早由英国生物学家理查德道金森在1976年提出, 它是一种基于达尔文进 化论的观点解释文化进化规律的新理论。道金森在其《自私的基因》中提到, “模因是一个文 化信息单位，那些不断得到复制和传播的语言、文化习俗、观念或社会行为等都属于模因。 模因可以看作是复制因子（replicator）, 也可以看作是文化进化单位。”既然语言属于一种典 型的复制因子, 那么是否可以运用模因理论的原理, 利用模因的特性, 应用于作文写作练习 的过程中, 来帮助学生获得语言的模因并运用到写作考试中呢?

基于上述研究目的, 笔者运用实证研究的方法, 探讨模因式英语教学模式对非英语专业学 生的四级写作的影响。重点将探讨以下三个问题：（1）与本校英语教师带有个人习惯和特点 的传统四级写作的教学模式相比, 模因式教学模式能否帮助学生提高四级写作水平? (2) 模 因式教学模式能够在哪些方面帮助学生提高写作能力? (3) 模因式教学模式是否能被本校学 生接受，此模式有何局限性以及在具体实施过程中存在哪些问题?

\section{3. 实验过程}

\section{实验前}

在实验前邀请50名学生参加四级写作测试, 要求他们在30分钟时间内独立完成一篇120字 左右的英语作文, 作文类型和话题以四级考试真题为参照来设计, 以模拟四级作文考试的方 式来实施。对作文进行评阅和打分，评分标准按照四级考试标准执行。

实验中

在模因理论中，模因在复制和传播中通常被认为经历几个不同阶段，其中包括同化 (assimilation)、记忆期(retention)、表达期(expression)和传播期(transmission)。在实验中，也 将此规律应用到写作模因教学的具体实施中。

同化期:

呈现给学生四级水平教材中的高频词汇和常用句型。在课堂上套用相似语境让学生反复加 深理解。此阶段时间较长, 需要教师与学生的耐心与合作。教师可提供给学生一些固定情境 让学生来套用表达, 加强学生的表达意识。

记忆期:

此阶段是时间最长并且最枯燥的阶段。这一阶段的主要目的是帮助学生内化吸收已学内 容。做到熟练背诵和理解。为了使学生能够保持学习兴趣, 教师可采取灵活多样的教学手段。 例如，小组讨论，单词接龙，故事接龙，课堂随机抽查等。

表达期:

此阶段是学生由模因记忆向模因异形传递的重要阶段。在这一阶段, 主要提高学生在变换 的语境下使用不同表达形式, 追求表达的多样性, 避免重复。教师可以安排学生进行同义转 换、句子变形、改编文章以及讲故事等方式，锻炼学生创造性使用语言的能力。 
传播期:

基于以上阶段的训练, 学生可以开始进入应用和检验阶段。教师可以选择重点话题有针对 地让学生进行命题写作练习, 强化和巩固重点强势模因, 与此同时, 还可以辅助进行段落翻 译的练习。此模因写作教学持续进行了两个月, 在开设的每周两节的写作课上进行。

实验后

培训后, 要求学生在给定时间内修改试验前所完成的作文或者重新写作。对前后两次的作 文进行数据分析, 并且利用CLEC语料库进行分类对比。

\section{4. 实验结果}

实验后, 笔者将学生的作文进行前后分析, 运用Range GSL AWL软件的三级词表进行词汇 分布对比分析, 结果如下:

表1 词汇分布对比分析

\begin{tabular}{|l|c|c|c|c|}
\hline & \multicolumn{2}{|c|}{ 形符数量/比例 $(\%)$} & \multicolumn{2}{|c|}{ 类符数量/比例 (\%) } \\
\hline & 实验前 & 实验后 & 实验前 & 实验后 \\
\hline 一级词汇 & $8527 / 80.23$ & $8834 / 80.02$ & $723 / 54.29$ & $750 / 53.20$ \\
\hline 二级词汇 & $1007 / 9.28$ & $1126 / 9.04$ & $153 / 11.72$ & $164 / 10.96$ \\
\hline 三级词汇 & $356 / 3.20$ & $408 / 4.10$ & $158 / 11.97$ & $173 / 14.03$ \\
\hline
\end{tabular}

表 2 常用词块的 $t$ 检验结果

\begin{tabular}{|c|c|c|c|c|c|c|}
\hline & $\mathrm{N}$ & 词块数 & 均值 & 标准差 & $\mathrm{t}$ & $\mathrm{p}$ \\
\hline 实验前 & 50 & 157 & 2.3781 & 1.32874 & & \\
\hline 实验后 & 50 & 218 & 3.1082 & 2.00184 & & \multirow{2}{*}{2.032} \\
\hline
\end{tabular}

利用中国学习者英语语料库(CLEC)的非英语专业大学生语料库提取常用词块100个, 再利 用语料库检索软件通过计算，得出关于常用词块的 $\mathrm{t}$ 检验结果如下:

通过t检验得出的前后作文成绩对比结果如下: 
表3 作文成绩对比结果

\begin{tabular}{|c|c|c|c|c|c|}
\hline & $\mathrm{N}$ & 均值 & 标准差 & $\mathrm{t}$ & $\mathrm{p}$ \\
\hline 实验前 & 50 & 8.4701 & 1.02183 & & \multirow{2}{*}{5.198} \\
\hline 实验后 & 50 & 9.0381 & 1.12850 & & 0.019 \\
\hline
\end{tabular}

\section{5. 结语}

通过上述数据, 可以得出以下结论。通过模因写作教学法, 学生们的词汇量以及在写作中 所使用的词汇级别都有所提高。同时, 通过复制传播的模因特点, 学生们使用词块和常用短 语的频率显著提高，最后作文成绩也有显著提高。

作为文化的组成部分，语言本身就是一种模因复合体，不同的复合体以框架的形式储存在 大脑里, 随时可能被激活。语言的使用和选择的过程就是各种模因相互竞争的过程。而生命 力强的高质量模因在竞争中才能存活下来, 即成功地被复制和传播。在英语教学过程, 被教 师反复强化的词汇与表达成为强势模因, 慢慢成为学生写作中的主要构成成分。加以合理利 用, 学生的写作成绩出现了明显提高。

模因教学模式合理利用模因的传播属性, 应用于写作教学当中, 对于学生写作成绩的提高 具有一定的帮助作用。在实际教学中, 教师们可以借鉴这种教学模式, 结合学生自身水平和 特点, 设计合理的教学手段和形式多样的教学活动, 帮助学生提高写作成绩和水平。

当然, 在实验后期, 笔者也发现模因教学模式在写作初期的教学效果会更加明显, 满足了学 生短期提高成绩的需要。但是从长远角度来看, 尤其是针对创造性写作, 模因教学模式并未 显现出具有较强说服力的效果。在这一方面, 仍然需要对模因理论以及写作本质进行更近一 步的研究和探讨。

\section{References}

[1] Blackmore, S. The Meme Machine. Oxford University Press, 1999.

[2] Chen Linxia. Meme and College English Writing Teaching. Foreign Language Research. Vol.1, p.89, 2008.

[3] Dawkins, R. The Selfish Gene. Oxford University Press, 2006.

[4] Gui Shichun and Yang Huizhong. Chinese Learner English Corpus. Shanghai Foreign Language Education Press, 2003.

[5] He Ziran. Meme in Language. Linguistic Sciences. Vol.6, pp.54-58, 2005.

[6] He Ziran, Xie Chaoqun, and Chen Xinren. New Developments in Pragmatics: Relevance, Adaptation and Memetics. Shanghai Foreign Language Education Press, 2007. 